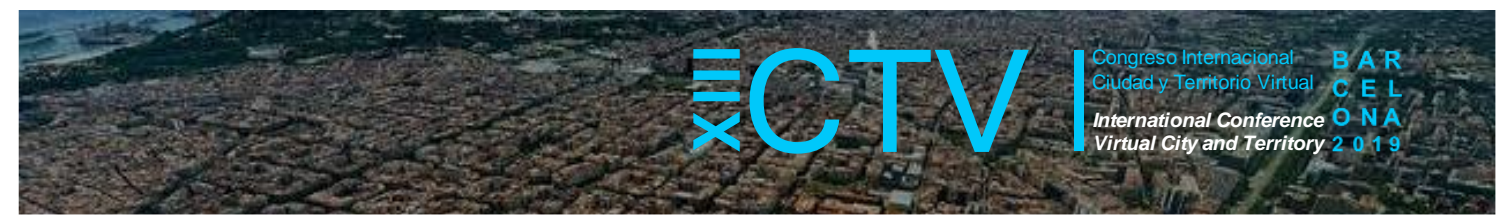

\title{
EPISODIOS DE INTENSIDAD EXTREMA DE PRECIPITACIÓN EN BARCELONA. RIESGO DE INUNDACIONES SÚBITAS
}

\author{
Martínez, Maria Dolors ${ }^{1 *}$; Lana, Xavier ${ }^{2}$; Serra, Carina ${ }^{3}$; Casas-Castillo, M. Carmen ${ }^{4}$; \\ Rodríguez-Solà, Raül ${ }^{5}$; Roca, Josep ${ }^{6}$; Arellano, Blanca ${ }^{7}$ y Biere, Rolando ${ }^{8}$
}

Remisión inicial: 2019-05-19; Remisión definitiva: 2019-10-15; Publicación: 2019-12-21

Citación: Martínez, M. D. et al. (2019). Episodios de intensidad extrema de precipitación en Barcelona. Riesgo de inundaciones súbitas. En XIII CTV 2019 Proceedings: XIII International Conference on Virtual City and Territory: "Challenges and paradigms of the contemporary city": UPC, Barcelona, October 2-4, 2019. Barcelona: CPSV, 2019, p. 8315. E-ISSN 2604-6512. DOI http://dx.doi.org/10.5821/ctv.8315

\section{Resumen}

Uno de los rasgos principales de las áreas urbanas es el predominio de las superficies impermeables (suelos sellados). Dependiendo de su densidad y extensión, el sellado de los suelos limita o impide las funciones naturales del suelo, puesto que reduce su capacidad potencial de infiltración del agua y, con ello, la regulación de la escorrentía aumentando el riesgo de inundaciones. En el caso de precipitaciones muy intensas en áreas urbanas con una elevada proporción de superficies impermeables, el sistema de alcantarillado puede no ser suficiente para hacer frente al exceso de agua de lluvia y, por lo tanto, se pueden producir inundaciones súbitas. En muchas zonas de Europa, la extensión de la urbanización y su densificación han incrementado notablemente la proporción de superficies impermeables (European Environment Agency, 2016). En la conurbación de Barcelona, la mayor parte de las áreas tienen una proporción de suelos sellados del $60-80 \%$ y en la ciudad de Barcelona, se supera el $80 \%$ (fuente: European Environment Agency, GMES/Copernicus). En esta zona los episodios de precipitación intensa son relativamente frecuentes, especialmente a finales de verano y durante el otoño, y algunos de ellos han dado lugar a inundaciones súbitas. En este trabajo se analizan los registros de una red de 23 estaciones de intensidad de precipitación instaladas en la ciudad de Barcelona. La base de datos consta de series de valores de precipitación acumulada a intervalos de 5 minutos, lo cual proporciona una medida de la intensidad de precipitación ( $\mathrm{mm} / 5$ minutos). El periodo de registro considerado abarca 16 años (1994-2009). Para los registros de más de 60 minutos de duración y de más de 25 mm (67 episodios, 499 registros) se han calculado el total de precipitación acumulada, la intensidad media y la intensidad máxima. Finalmente, se han estudiado los que exceden al mismo tiempo, el percentil del $95 \%$ de estas tres variables. Con ello, se han obtenido 29 registros extremos que podrían dar lugar a situaciones de inundación súbita. Estos registros extremos se agrupan en siete episodios distintos, ocurridos entre finales de julio y octubre. Una buena parte de estos episodios extremos se produce por procesos de origen convectivo a escala local, así como por advecciones del este. Los siete episodios extremos analizados, provocaron importantes afectaciones en las comunicaciones viales, averías en los semáforos y en las líneas telefónicas, así como interrupción del servicio de algunas líneas del metro, del aeropuerto y de la red ferroviaria. Como consecuencia del cambio climático y el calentamiento global, es probable que aumente la frecuencia de los episodios de precipitación muy intensa. El desarrollo de políticas dirigidas a limitar o reducir la proporción de suelos sellados en las áreas urbanas sería un elemento clave para mitigar, en lo posible, este riesgo de inundaciones súbitas.

\section{Abstract}

One of the main features of urban areas is the prevalence of impervious surfaces (sealed soils). Depending on its density and extent, soil sealing restricts natural soil functions, since it reduces water infiltration capacity and run-off regulation, thus increasing flash flooding risk. In the case of heavy rainfalls in urban areas with high imperviousness ratio, the sewage system could no longer be able to cope with the excess of rainwater and then pluvial floods develop.

\footnotetext{
1 Departamento de Física, ETSBA, UPC, https://orcid.org/0000-0001-8200-183X; ${ }^{2}$ Departamento de Física, ETSEIB, UPC, https://orcid.org/0000-0002-3298-9234; ${ }^{3}$ Departamento de Física, ETSEIB, UPC, https://orcid.org/0000-00033803-2414; ${ }^{4}$ Departamento de Física, ESEIAAT, https://orcid.org/0000-0002-7507-6195; ${ }^{5}$ Departamento de Física, ETSEIB, UPC, https://orcid.org/0000-0002-9623-894X; ${ }^{6}$ Centro de Política del Suelo y Valoraciones, CPSV, TA, UPC, https://orcid.org/0000-0003-3970-6505; ${ }^{7}$ Centro de Política del Suelo y Valoraciones, CPSV, TA, UPC, https://orcid.org/0000-0001-7128-3667 y ${ }^{8}$ Centro de Política del Suelo y Valoraciones, CPSV, TA, UPC, https://orcid.org/0000-0003-1872-7104. *Correo de contacto: dolors.martinez@upc.edu.
} 


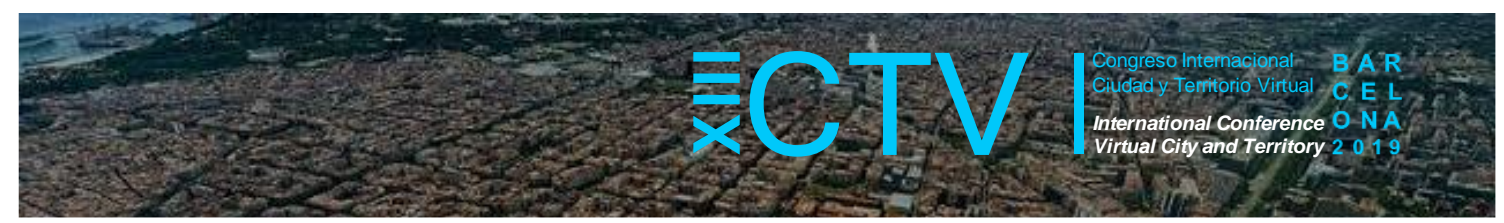

In many European zones, urban spread and densification have increased the impervious surface ratio (European Environment Agency, 2016). In Barcelona conurbation, most areas have high fractions of sealed soils (60-80\%) and in the city, this fraction exceeds $80 \%$ (source: European Environment Agency, GMES/Copernicus). Heavy rainfall episodes are relatively frequent in this area, especially in autumn, and some of them have resulted in flash floods. In this study, rainfall intensity records from an urban network of 23 stations are analysed. The database consists of series of rain amounts collected at 5 -minute intervals, which provides a measure of rainfall intensity ( $\mathrm{mm} / 5$ minutes). The recording period encompasses 16 years (1994-2009). For all the records with durations greater than 60 minutes and total amounts greater than $25 \mathrm{~mm}$ (67 episodes, 499 records), the total rain amount, average intensity and maximum intensity are determined. We have selected the records exceeding, simultaneously, the $95^{\text {th }}$ percentile of these three variables, thus obtaining 29 extreme records, which could lead to pluvial flash flood events. These extreme records correspond to seven different episodes, occurred from the end of July to October. Most of these extreme episodes are generated by convective processes at local scale as well as by eastern advections. The seven extreme episodes caused important affectations in road communications, breakdowns at traffic lights and telephone lines, as well as interruption of the service of some suburban lines, the airport and the railway network. Because of climate change and global warming, the frequency of episodes of very heavy precipitation is likely to increase. The development of policies aimed at limiting or reducing the proportion of sealed soils in urban areas would be a key element to mitigate, as far as possible, this risk of flash flooding.

Palabras Clave: intensidad de precipitación; episodios extremos; superficies impermeables; Barcelona

Keywords: rainfall intensity; extreme episodes; impervious surfaces; Barcelona

\section{Introducción}

Los procesos de urbanización modifican los usos del suelo. Como resultado del desarrollo urbano y de la construcción de infraestructuras, la superficie del suelo es recubierta por materiales impermeables. La Comisión Europea ha identificado el sellado de los suelos como uno de los principales procesos de degradación del suelo (EC, 2006), puesto que reduce, o incluso impide totalmente, sus funciones naturales de absorción, filtración y almacenamiento de agua (Prokop et al., 2011; EC-DGENV, 2012; EEA, 2014). El sellado de los suelos, pues, juega un papel primordial en el riesgo de inundaciones pluviales, dado que la reducción de su capacidad de infiltración del agua limita la regulación de la escorrentía. En el caso de precipitaciones muy intensas en áreas urbanas con una elevada proporción de superficies impermeables, el exceso de agua de escorrentía puede llegar a desbordar la capacidad de los sistemas de drenaje y dar lugar a inundaciones pluviales súbitas (Kaźmierczak and Cavan, 2011; EEA, 2015). Por otra parte, también merece ser mencionado que las superficies impermeables potencian el fenómeno de isla de calor urbana (UHI, urban heat island): las temperaturas en las áreas urbanas suelen ser más elevadas que en las zonas rurales próximas (Oke, 1982; Makar et al., 2006; Findell et al., 2007; Memon et al., 2009; Giannaros and Melas, 2012; Martínez et al., 2018, entre otros). En comparación con las áreas de vegetación, las superficies selladas absorben más radiación solar, alcanzan temperaturas más elevadas y liberan más lentamente el calor acumulado, lo cual es una de las causas del efecto UHI. Además, cuanto mayor es la proporción de superficies impermeables, menor es la evapotranspiración de la vegetación existente, lo cual contribuye a un mayor aumento de la temperatura del aire y de las superficies. Las ciudades con una elevada proporción de suelos impermeables y escasa presencia de vegetación urbana están expuestas a una mayor intensidad del efecto UHI (Heusinkveld et al., 2014).

En muchas zonas de Europa, la extensión de la urbanización y su densificación han provocado un incremento notable, en las últimas décadas, de la proporción de superficies impermeables (EEA, 2016). En la conurbación de Barcelona, la mayor parte de las zonas tienen una proporción de suelos sellados del $60-80 \%$ y en la propia ciudad, se supera el $80 \%$, como se observa en el mapa de la Figura 1, que muestra la densidad de superficies impermeables en 


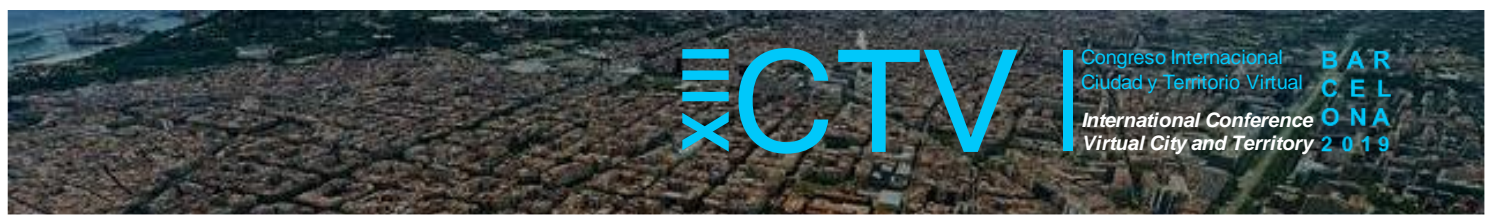

2015 (fuente: European Environment Agency, GMES/Copernicus). El régimen de precipitación

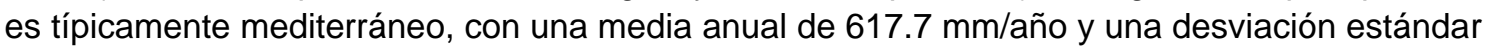
de 150.3 mm/año (Observatorio Fabra, 1917-2009). Los episodios de precipitación intensa son relativamente frecuentes y están asociados, usualmente, a advecciones del este en otoño y a procesos de origen convectivo a escala local en verano. Algunos de estos episodios presentan picos destacables de intensidad, con precipitación copiosa en intervalos de tiempo relativamente cortos, y han dado lugar a inundaciones pluviales (Casas et al., 2010; Lana et al., 2018, 2019a, 2019b).

Figura 1. Densidad de superficies impermeables en la conurbación de Barcelona en el año 2015

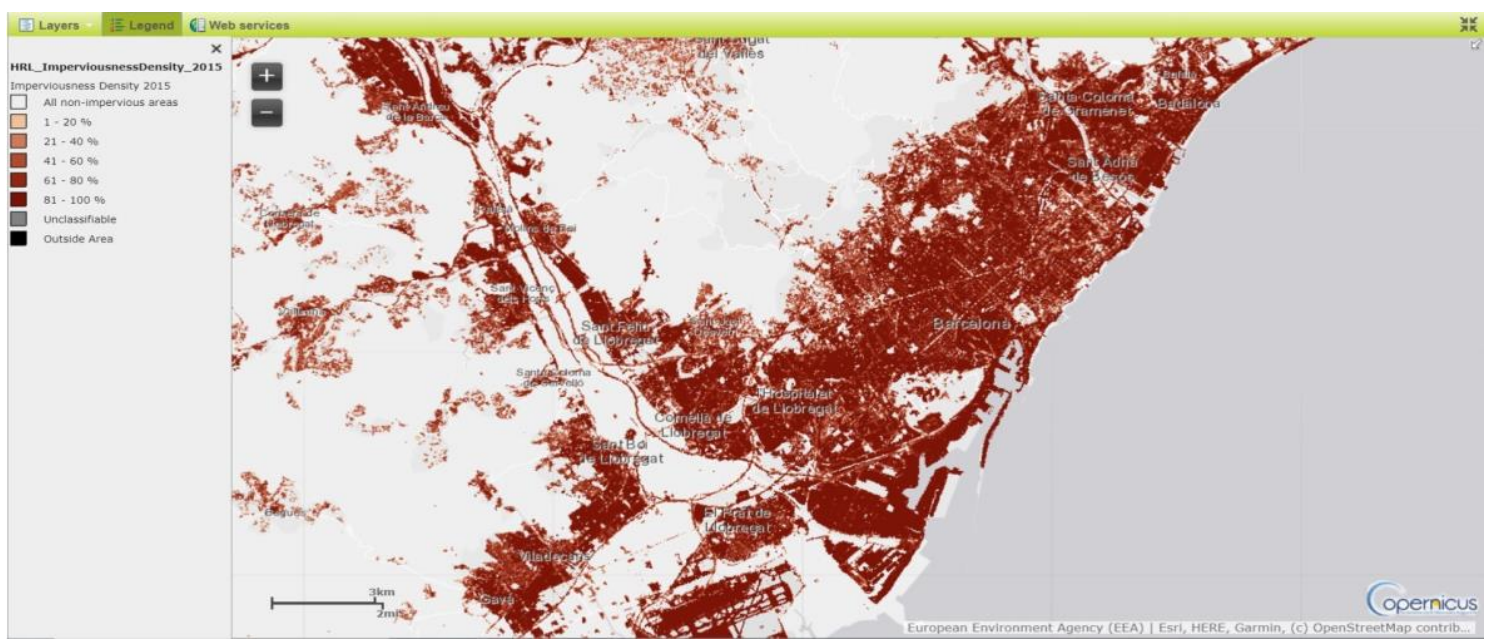

Fuente: European Environment Agency, GMES/Copernicus.

En este trabajo, se analizan los registros de intensidad de precipitación del periodo 1994-2009 en una red de estaciones instaladas en la ciudad de Barcelona. Con el objetivo de estudiar los episodios extremos de precipitación, que pueden dar lugar a inundaciones pluviales súbitas, se determina, para todos los registros del periodo analizado, el total de precipitación acumulada, la intensidad media y la intensidad máxima, y se seleccionan los registros que exceden, al mismo tiempo, el percentil del 95\% de estas tres variables. Estos registros extremos se agrupan en siete episodios, ocurridos entre finales de julio y octubre, que desbordaron la capacidad de los sistemas de drenaje y dieron lugar a inundaciones en la ciudad de Barcelona, que provocaron importantes afectaciones en las comunicaciones viales, averías en los semáforos y en las líneas telefónicas, así como interrupción del servicio de algunas líneas del metro, del aeropuerto y de la red ferroviaria.

\section{Base de datos y zona de estudio}

El área urbana de Barcelona (Figura 2), con una extensión aproximadamente de $100 \mathrm{~km}^{2}$, se caracteriza por ser una ciudad costera mediterránea limitada en su parte interior por la Sierra de Collserola, cuya máxima elevación es de $512 \mathrm{~m}$. Al suroeste y el nordeste, el área metropolitana limita con los valles de los ríos Llobregat y Besós. También presenta alguna pequeña colina, como la de Montjuïc, de unos $150 \mathrm{~m}$ de altitud, cercana al puerto y con una pronunciada pendiente en su vertiente orientada al mar (Figura 3). Aunque las altitudes de estas montañas no son muy elevadas, las pendientes en algunos casos pueden ser considerables, lo que puede provocar inundaciones súbitas cuando las cantidades de precipitación son copiosas. 


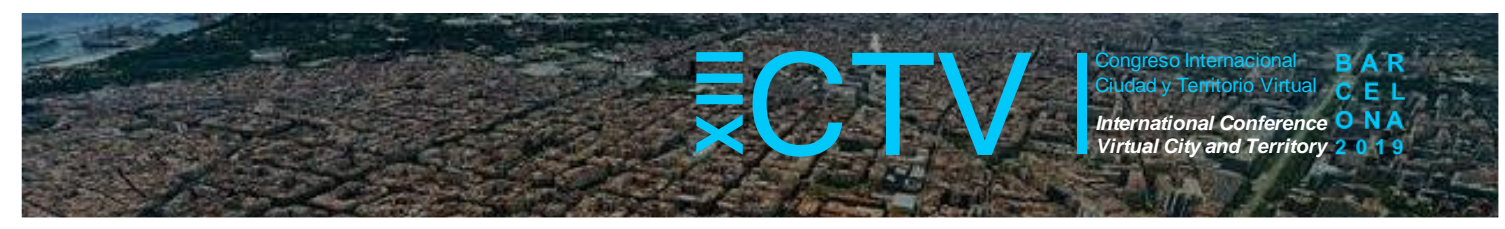

Figura 2. Ubicación geográfica del área metropolitana de Barcelona

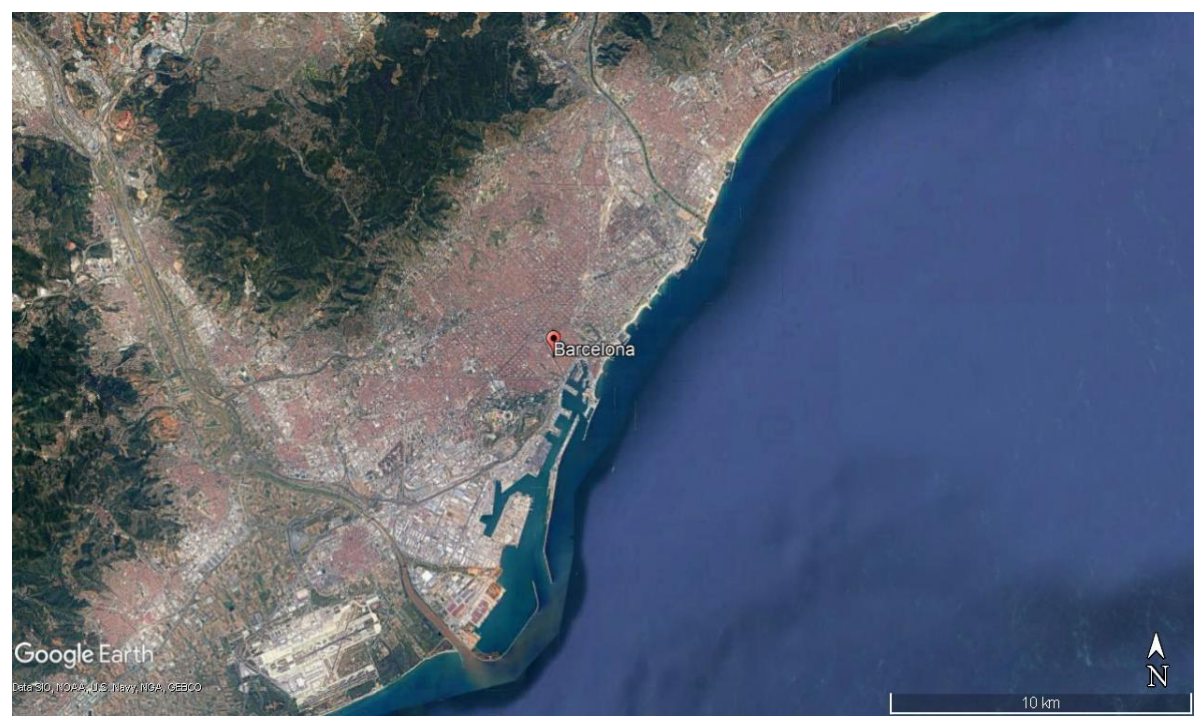

Fuente: Google Earth.

Figura 3. Topografía de Barcelona y distribución de las estaciones URBAN NETWORK - BARCELONA

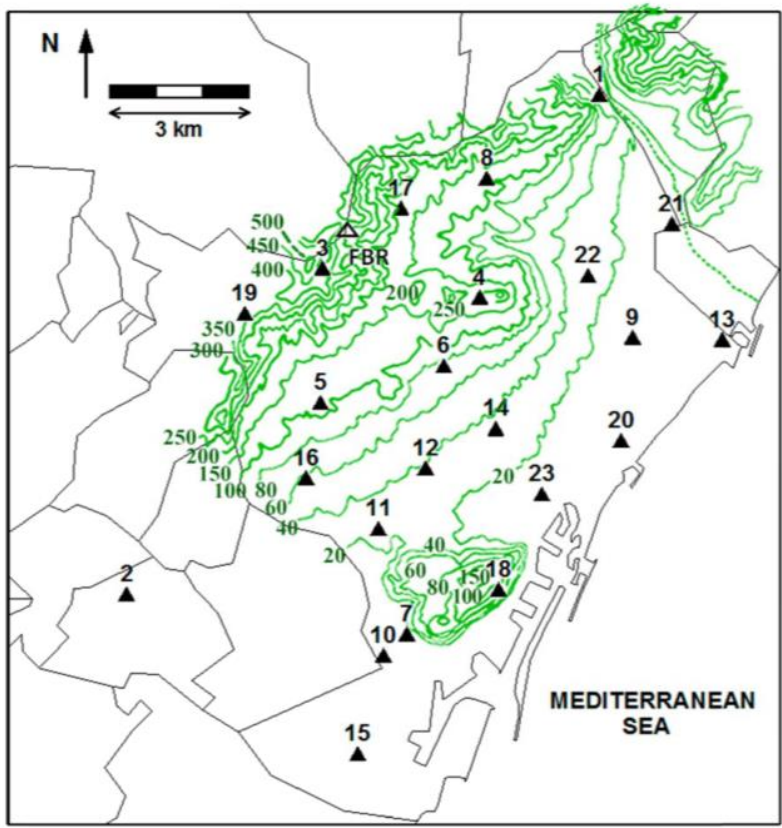

Fuente: Elaboración propia.

En la Figura 3 se muestra la topografía del área urbana de Barcelona y la distribución de las 23 estaciones. En ellas se registra la intensidad de precipitación con una resolución de $0.1 \mathrm{~mm} / 5$ min. La base de datos pluviométrica que se ha utilizado para este estudio incluye las intensidades de precipitación desde septiembre de 1994 hasta noviembre del 2009. En este trabajo, hemos optado por seleccionar los episodios que cumplen las siguientes condiciones (Lana et al., 2018, 2019a,2019b): 


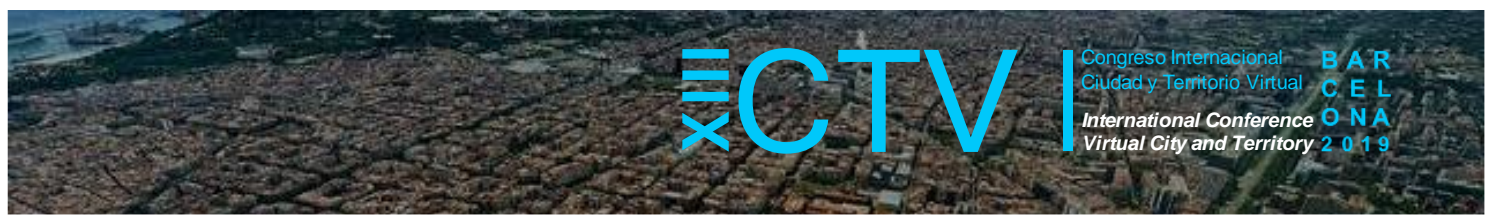

- Los episodios están formados por registros de precipitación que pueden incluir intervalos sin precipitación siempre y cuando estos intervalos no superen una hora. Cuando un intervalo de tiempo sin registro de lluvia supera los 60 minutos, dicho intervalo separa dos episodios.

- Los episodios seleccionados son aquellos que tienen cantidades de precipitación con periodos de retorno mayores o iguales a 1 año, en al menos uno de los intervalos de tiempo de entre 5 minutos y 24 horas analizados en Casas et al. (2010).

- Las cantidades de precipitación de los episodios deben ser iguales o superiores a 25 $\mathrm{mm}$, ya que estos son los que se consideran potencialmente peligrosos (Huff, 1967).

- La duración del episodio debe ser igual o superior a 60 min.

Las dos últimas restricciones relacionadas con la duración del registro y la cantidad de precipitación se han impuesto para tener en cuenta las características de los episodios potencialmente peligrosos según la Agencia Española de Meteorología (AEMET) y el Servei Meteorològic de Catalunya (SMC). Si sólo se aplican las dos primeras restricciones se obtienen un total de 188 episodios con duraciones predominantes entre 3 y 7 horas, y algunos de ellos de hasta 24 horas. Los valores más frecuentes de precipitación total acumulada en un episodio individual son de 5 a $25 \mathrm{~mm}$ y se alcanzan máximos de hasta $140 \mathrm{~mm}$. Las intensidades máximas de precipitación varían entre 10 y $19 \mathrm{~mm} / 5$ minutos (Lana et al. 2018). Sin embargo, al tener en cuenta las cuatro restricciones, trabajamos con 67 episodios que incluyen 499 registros 5-min de las diferentes estaciones de la red pluviométrica.

\section{Metodología y resultados}

En la Tabla 1 se muestran los valores de diferentes magnitudes pluviométricas de las 23 estaciones de la red y de los 67 episodios escogidos, como la cantidad de precipitación promedio $\langle R>$, y su desviación, la duración promedio $\langle L>$ y su desviación, la intensidad máxima 5-min promedio $<I_{\max }>$ y su desviación, la intensidad máxima 5 -min $I_{M}$, la fecha en que fue registrada y la cantidad total $R\left(I_{M}\right)$ para el episodio que incluye la máxima intensidad $I_{M}$. Las cantidades de precipitación están expresadas en mm y la duración en minutos.

El episodio del 14/09/1999 aparece en 11 de las 23 estaciones como el que registró la intensidad 5-min más alta. Dicho episodio ya ha sido comentado en anteriores publicaciones como uno de los más intensos de los últimos años (Casas et al. 2010). También la prensa local se hizo eco del fenómeno. Hubo inundaciones en la Plaza Cerdà y en la Plaza de España, la Ronda del Mig y el metro. El aeropuerto y los trenes de Cercanías se paralizaron durante la mañana, los bomberos de la Generalitat realizaron 370 salidas, las riadas de los pueblos costeros vecinos arrastraron coches, las líneas de teléfono de las policías locales se colapsaron y varias personas tuvieron que ser rescatadas de sus coches 0 de sus viviendas (La Vanguardia, 15/09/1999).

Tabla 1. Valores relacionados con las cantidades de precipitación e intensidades 5min de las 23 estaciones de la red pluviométrica de Barcelona

\begin{tabular}{|c|c|c|c|c|c|c|c|c|c|}
\hline Estación & $<\mathbf{R}>$ & $\boldsymbol{\sigma}(\mathbf{R})$ & $<\mathbf{L}>$ & $\boldsymbol{\sigma}(\mathbf{L})$ & $\left\langle\mathbf{I}_{\max }>\right.$ & $\boldsymbol{\sigma}\left(\mathbf{I}_{\max }\right)$ & $\mathbf{I}_{\mathbf{M}}$ & $\mathbf{F e c h a}\left(\mathbf{I}_{\mathbf{M}}\right)$ & $\mathbf{R}\left(\mathbf{I}_{\mathbf{M}}\right)$ \\
\hline 1 & 45.0 & 21.8 & 446.8 & 324.3 & 4.7 & 2.6 & 10.0 & $07 / 09 / 2005$ & 34.5 \\
\hline 2 & 45.4 & 23.1 & 435.6 & 340.4 & 5.0 & 2.7 & 12.4 & $14 / 09 / 1999$ & 60.7 \\
\hline 3 & 51.1 & 20.7 & 568.9 & 394.2 & 4.2 & 2.6 & 9.0 & $14 / 09 / 1999$ & 51.0 \\
\hline 4 & 57.7 & 27.8 & 516.8 & 293.7 & 6.1 & 3.0 & 12.3 & $15 / 07 / 2001$ & 66.7 \\
\hline 5 & 47.6 & 23.3 & 448.2 & 318.9 & 5.6 & 3.3 & 15.5 & $14 / 09 / 1999$ & 67.4 \\
\hline
\end{tabular}




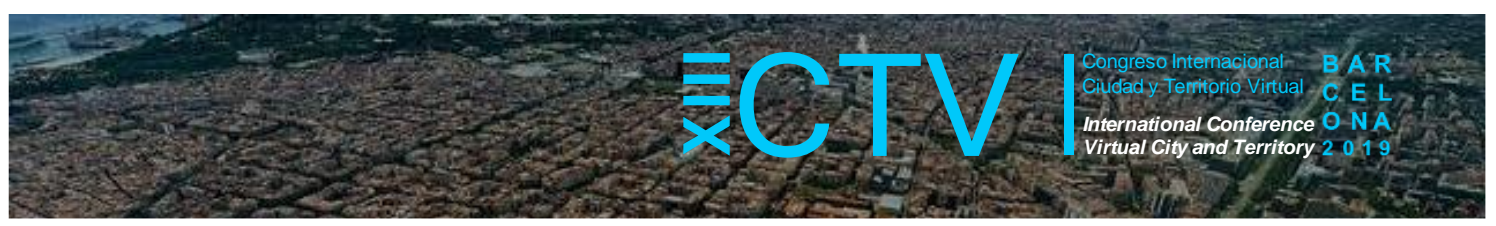

\begin{tabular}{|c|c|c|c|c|c|c|c|c|c|}
\hline 6 & 48.1 & 18.9 & 461.9 & 343.4 & 5.2 & 3.6 & 19.6 & $21 / 09 / 1995$ & 91.9 \\
\hline 7 & 43.9 & 22.1 & 416.5 & 334.2 & 5.9 & 3.1 & 12.4 & $14 / 09 / 1999$ & 56.8 \\
\hline 8 & 43.6 & 18.7 & 477.2 & 350.7 & 4.9 & 2.6 & 10.2 & $02 / 08 / 2005$ & 30.9 \\
\hline 9 & 51.7 & 21.4 & 516.8 & 342.8 & 4.8 & 2.6 & 11.4 & $14 / 09 / 1999$ & 46.4 \\
\hline 10 & 45.6 & 21.7 & 440.8 & 350.5 & 6.0 & 3.4 & 13.9 & $26 / 08 / 2002$ & 40.5 \\
\hline 11 & 55.5 & 23.3 & 463.3 & 328.5 & 6.5 & 3.4 & 14.0 & $14 / 09 / 1999$ & 58.2 \\
\hline 12 & 44.3 & 24.6 & 339.2 & 239.7 & 5.9 & 2.5 & 12.0 & $17 / 10 / 2008$ & 28.3 \\
\hline 13 & 53.4 & 27.1 & 581.8 & 427.3 & 4.7 & 2.6 & 10.1 & $17 / 10 / 1999$ & 21.4 \\
\hline 14 & 51.7 & 30.4 & 497.3 & 312.5 & 5.5 & 2.9 & 12.3 & $14 / 09 / 1999$ & 51.0 \\
\hline 15 & 43.7 & 20.7 & 455.0 & 325.2 & 5.4 & 2.8 & 12.3 & $14 / 09 / 1999$ & 53.9 \\
\hline 16 & 44.6 & 15.1 & 427.1 & 337.9 & 5.4 & 2.9 & 11.0 & $23 / 10 / 1997$ & 32.6 \\
\hline 17 & 54.2 & 25.2 & 448.7 & 340.2 & 5.8 & 3.1 & 11.7 & $17 / 11 / 1996$ & 29.0 \\
\hline 18 & 51.4 & 27.4 & 460.4 & 326.4 & 5.6 & 3.2 & 13.0 & $14 / 09 / 1999$ & 51.0 \\
\hline 19 & 45.3 & 19.5 & 467.8 & 319.0 & 4.9 & 2.9 & 10.5 & $05 / 10 / 1998$ & 28.4 \\
\hline 20 & 46.4 & 25.4 & 424.1 & 324.1 & 6.4 & 2.9 & 12.8 & $14 / 09 / 1999$ & 47.2 \\
\hline 21 & 45.4 & 23.2 & 408.1 & 291.4 & 5.3 & 2.5 & 9.9 & $17 / 10 / 1999$ & 25.8 \\
\hline 22 & 53.7 & 29.5 & 418.1 & 347.9 & 6.0 & 2.6 & 11.5 & $14 / 09 / 1999$ & 48.6 \\
\hline 23 & 56.8 & 24.5 & 514.6 & 412.6 & 5.4 & 3.5 & 11.3 & $24 / 08 / 1995$ & 94.2 \\
\hline
\end{tabular}

Fuente: Elaboración propia.

El primer gráfico de la Figura 4 (superior, izquierda) muestra la relación entre las cantidades totales de precipitación de los diferentes registros y su duración. Se puede observar que la cantidad total de precipitación y la duración del registro guardan una relación aproximadamente potencial. Las líneas de trazos discontinuos marcan las duraciones de 60 minutos y cantidades de $25 \mathrm{~mm}$. Por lo tanto, los puntos que están en el cuadrante superior derecho serían los escogidos para hacer el estudio. En el segundo gráfico (superior, derecha), se muestra la relación entre las cantidades totales de precipitación y el día del año en que se produjeron. Se observa que las cantidades de precipitación más importantes (mayores que $75 \mathrm{~mm}$ ) se producen entre finales de Julio y finales de noviembre, con un máximo de $136.5 \mathrm{~mm}$ que se registró el 12/10/2005. Una distribución similar a lo largo del año se observa en los otros dos gráficos (parte inferior), para el valor máximo de la intensidad 5-min y para la intensidad promedio $R / L$, calculada como el cociente entre la cantidad total del registro $R$ y su duración $L$.

Figura 4. Relación entre la cantidad de precipitación y la duración del registro y distribución de las cantidades de precipitación
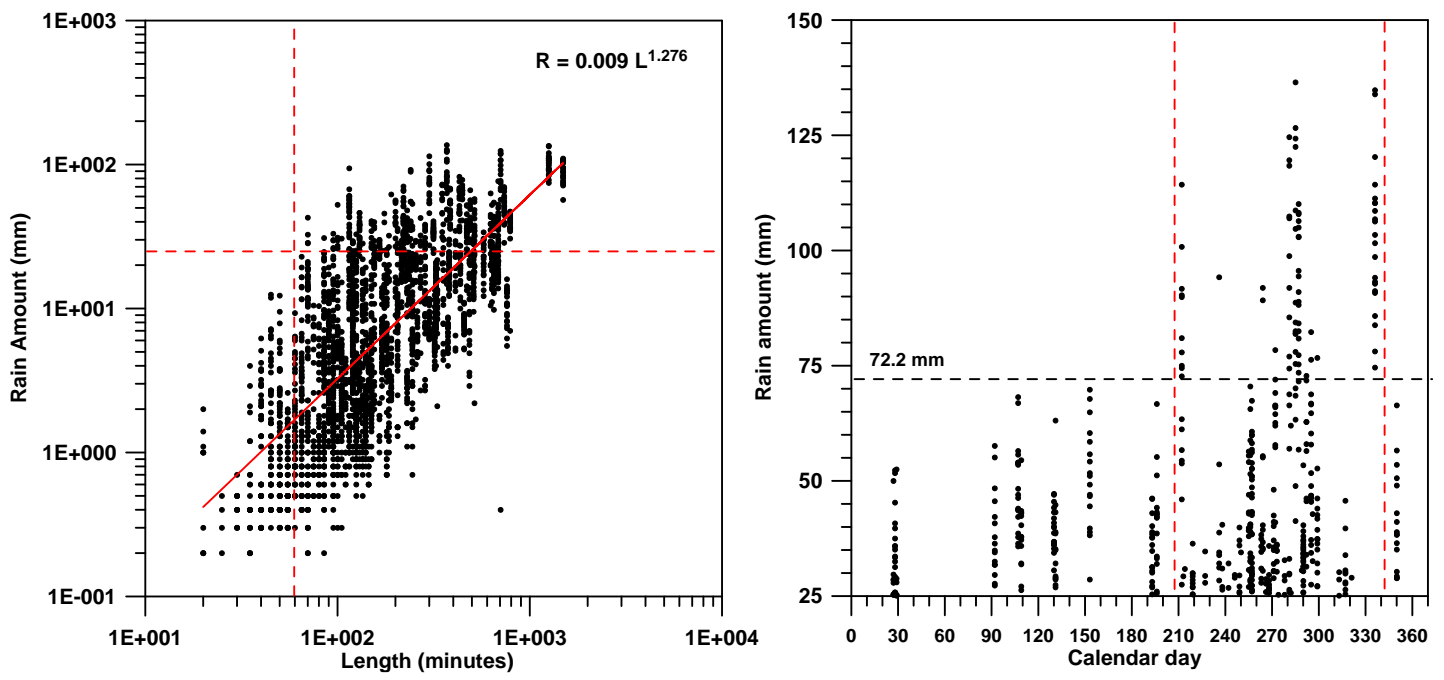

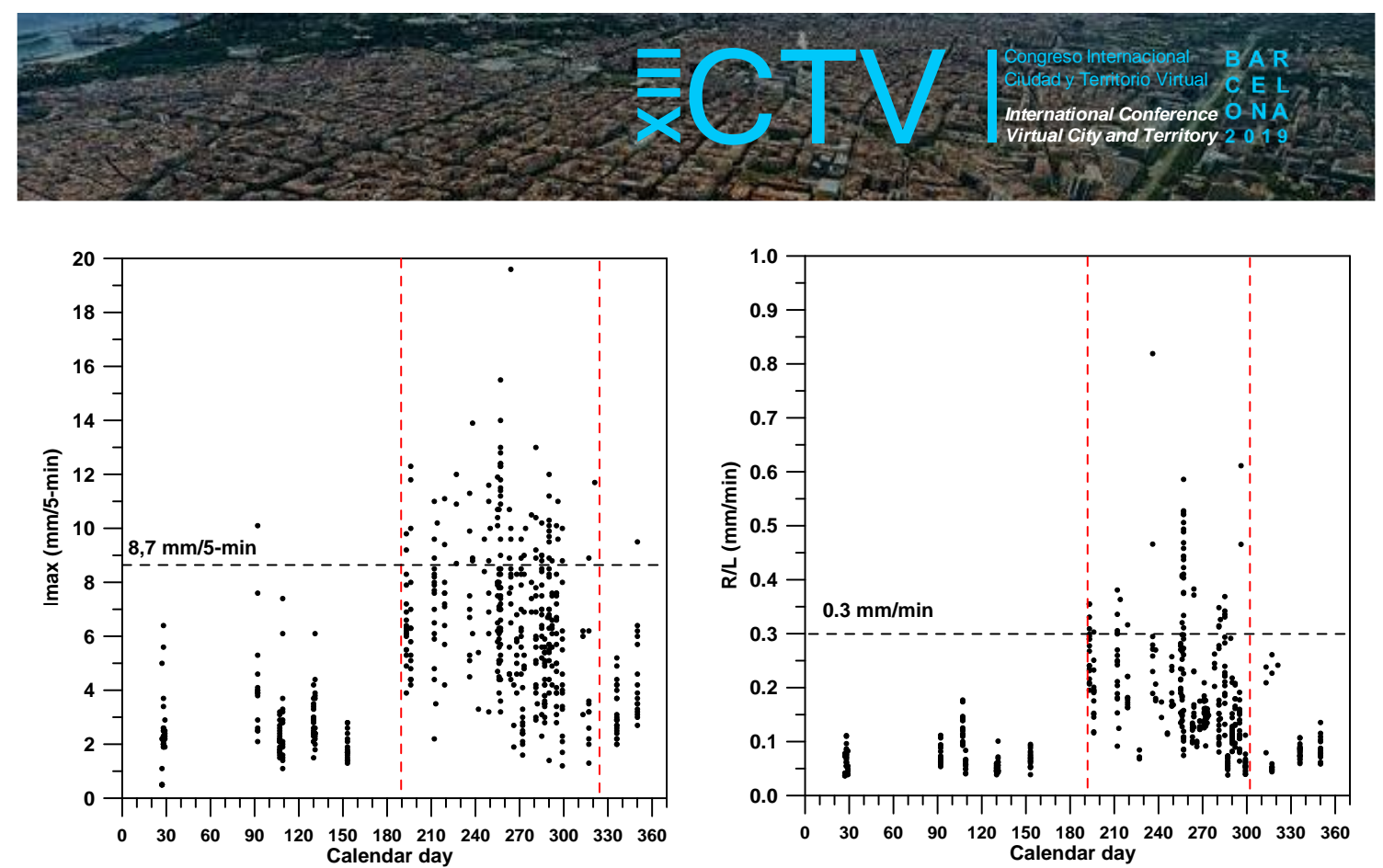

Nota: La intensidad máxima 5-min y la intensidad promedio según el día del año. Fuente: Elaboración propia.

En la Figura 5 se muestran cuatro ejemplos de registros que presentan distribuciones diferentes de la intensidad 5-min. Analizar el tipo de distribución es interesante desde el punto de vista de la prevención de riadas y del diseño de la red de colectores y desagües o de reducción de suelos sellados. El grueso de la precipitación tiene una duración entre los 20 y 50 minutos en los tres registros de verano. En el ejemplo de 12/10/2005, ya entrado el otoño, la duración es mayor. En el primer ejemplo se aprecian tres picos de intensidad, con intermitencias o intervalos sin lluvia, siendo el último pico el más destacado. Todo hace pensar que corresponden a tres células convectivas, típicas de finales de verano. El máximo puede aparecer tanto al principio, como a la mitad o al final del episodio. El registro de la estación número 14 es el que presenta el total más alto con $136.5 \mathrm{~mm}$ (12/10/2005). En Lana et al. (2019b) se ha realizado una clasificación de los diferentes registros en función de su distribución temporal, obteniéndose 10 grupos (clusters) con diferentes perfiles de intensidades de lluvia (mm/5-min).

Figura 5. Distribución temporal de la intensidad 5-min de la precipitación de algunos ejemplos de precipitación extrema
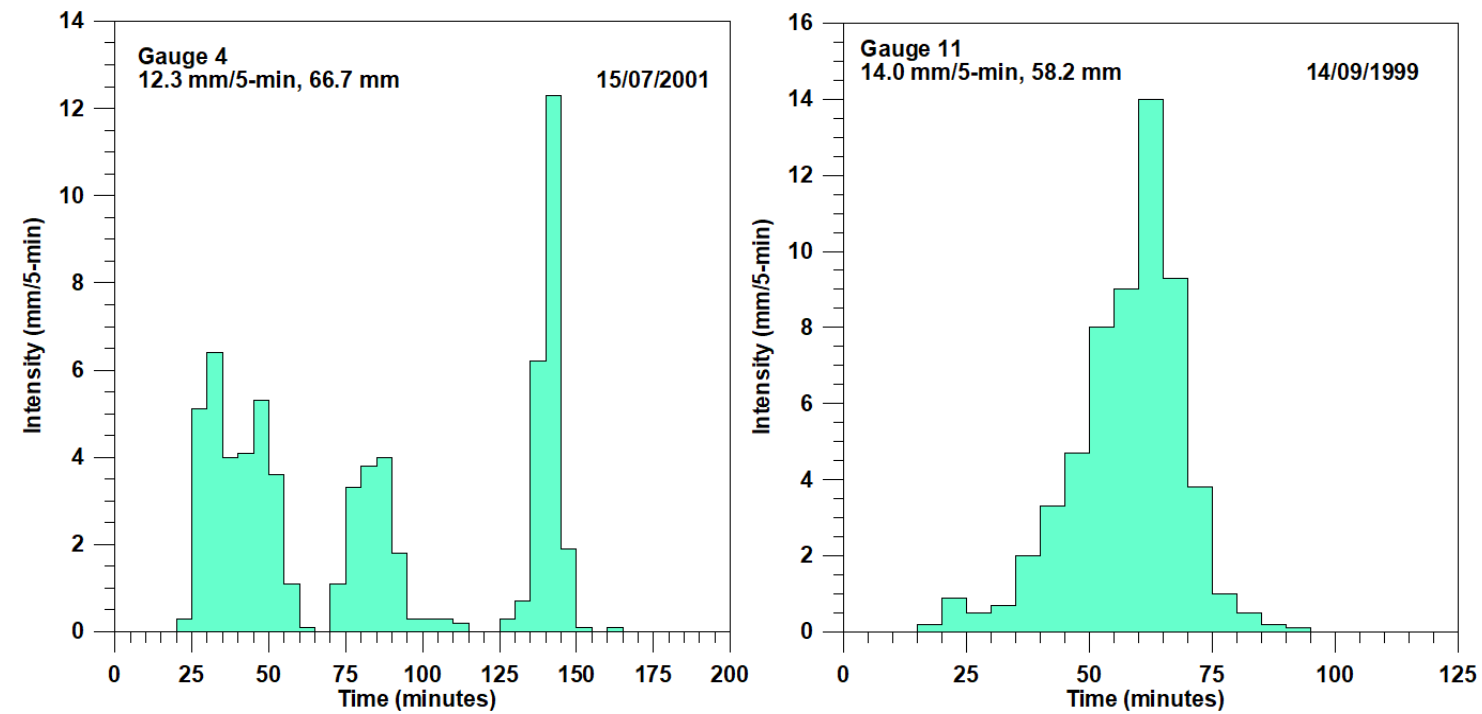

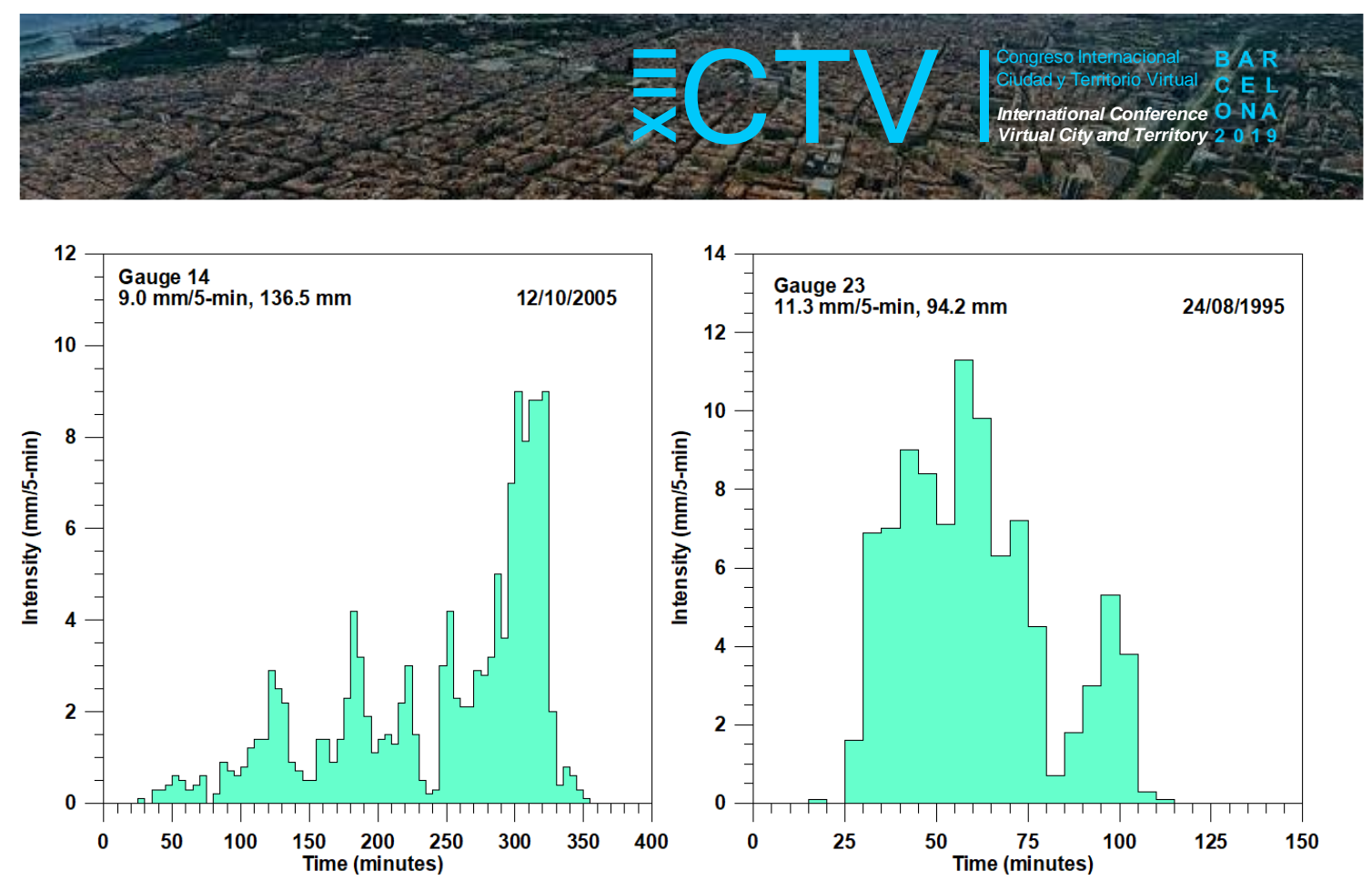

Fuente: Elaboración propia.

En la Tabla 2 se muestran los 29 registros pertenecientes a los 7 episodios que exceden el percentil del $95 \%$ en la cantidad total de precipitación, la intensidad máxima 5-min y la intensidad media R/L. Destaca el episodio del 12/10/2005 por registrarse cantidades de más de $100 \mathrm{~mm}$ en 5 estaciones. Por otro lado, resaltan las estaciones 11 y 17 por ser las que aparecen en la tabla en 3 episodios distintos. También hay que mencionar que en las dos ocasiones en que aparece la estación 14, las cantidades superan los $100 \mathrm{~mm}$. En futuros análisis, en los que se ampliará el periodo de estudio hasta el 2019, se podrá confirmar si dichas estaciones están ubicadas en zonas que registran sistemáticamente precipitaciones más extremas.

Tabla 2. Registros que superan el percentil del $95 \%$

\begin{tabular}{|c|c|c|c|c|c|}
\hline Fecha & Estación & $I_{\max -5 \min }$ & Total(mm) & Duración(min) & $\mathbf{R} / \mathbf{L}$ \\
\hline $24 / 08 / 1995$ & 23 & 11.3 & 94.2 & 115 & 0.82 \\
\hline $21 / 09 / 1995$ & 6 & 19.6 & 91.9 & 240 & 0.38 \\
\hline $21 / 09 / 1995$ & 11 & 9.6 & 55.1 & 240 & 0.23 \\
\hline $21 / 09 / 1995$ & 22 & 8.5 & 89.2 & 240 & 0.37 \\
\hline $21 / 09 / 1995$ & 23 & 10.0 & 55.4 & 240 & 0.23 \\
\hline $14 / 09 / 1999$ & 2 & 12.4 & 60.7 & 115 & 0.53 \\
\hline $14 / 09 / 1999$ & 5 & 15.5 & 67.4 & 115 & 0.59 \\
\hline $14 / 09 / 1999$ & 7 & 12.4 & 56.8 & 115 & 0.49 \\
\hline $14 / 09 / 1999$ & 11 & 14.0 & 58.2 & 115 & 0.51 \\
\hline $14 / 09 / 1999$ & 16 & 8.3 & 56.2 & 115 & 0.49 \\
\hline $14 / 09 / 1999$ & 17 & 10.9 & 59.9 & 115 & 0.52 \\
\hline $15 / 07 / 2001$ & 4 & 12.3 & 66.7 & 220 & 0.30 \\
\hline $15 / 07 / 2001$ & 10 & 11.8 & 55.2 & 220 & 0.25 \\
\hline $31 / 07 / 2002$ & 3 & 8.5 & 74.5 & 300 & 0.25 \\
\hline $31 / 07 / 2002$ & 5 & 11.0 & 114.3 & 300 & 0.38 \\
\hline $31 / 07 / 2002$ & 11 & 7.9 & 72.7 & 300 & 0.24 \\
\hline $31 / 07 / 2002$ & 14 & 8.2 & 100.8 & 300 & 0.30 \\
\hline $31 / 07 / 2002$ & 16 & 8.9 & 81.0 & 300 & 0.27 \\
\hline $31 / 07 / 2002$ & 19 & 9.6 & 89.9 & 300 & 0.30 \\
\hline $31 / 07 / 2002$ & 20 & 8.0 & 91.7 & 300 & 0.31 \\
\hline $12 / 10 / 2005$ & 4 & 9.0 & 108.7 & 370 & 0.29 \\
\hline $12 / 10 / 2005$ & 14 & 9.0 & 136.5 & 370 & 0.37 \\
\hline $12 / 10 / 2005$ & 17 & 7.9 & 88.7 & 370 & 0.24 \\
\hline $12 / 10 / 2005$ & 20 & 8.8 & 122.5 & 370 & 0.33 \\
\hline
\end{tabular}




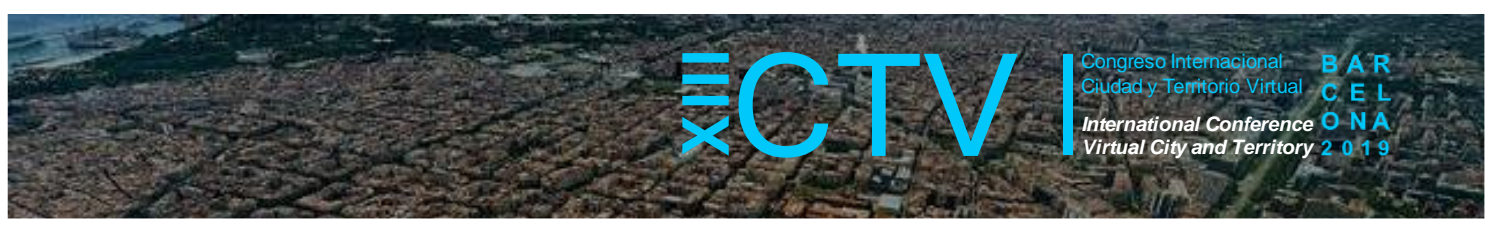

\begin{tabular}{|l|c|c|c|c|c|}
\hline $12 / 10 / 2005$ & 21 & 8.5 & 104.7 & 370 & 0.28 \\
\hline $12 / 10 / 2005$ & 22 & 10.2 & 124.3 & 370 & 0.36 \\
\hline $12 / 09 / 2006$ & 7 & 11.9 & 56.4 & 200 & 0.28 \\
\hline $12 / 09 / 2006$ & 15 & 10.4 & 55.5 & 200 & 0.28 \\
\hline $13 / 09 / 2006$ & 17 & 10.7 & 65.6 & 220 & 0.30 \\
\hline
\end{tabular}

Fuente: Elaboración propia.

Los mapas sinópticos en superficie y de altura geopotencial a $500 \mathrm{hPa}$ (Figura 6) revelan, en la mayor parte de los casos, que los gradientes de presión en superficie (isolíneas blancas) son muy débiles, mientras que a $500 \mathrm{hPa}$ (zonas en distintos colores) se observa un surco, con el área de Barcelona en su parte delantera. Esta situación, junto con las elevadas temperaturas en superficie y el aporte de humedad del Mediterráneo, genera actividad convectiva, que puede dar lugar a episodios de precipitación copiosa en intervalos de tiempo relativamente cortos. Este tipo de episodios es el que puede provocar, con mayor probabilidad, una saturación de los sistemas de drenaje, lo cual daría lugar a inundaciones súbitas.

En el mapa de la Figura 6 se puede apreciar esta situación, correspondiente al episodio del $12 / 10 / 2005$, durante el cual cinco de las estaciones de la red registraron precipitaciones totales de más de $100 \mathrm{~mm}$, con un máximo de $136.5 \mathrm{~mm}$. Este episodio fue calificado por la prensa como 'el aguacero de la década' (La Vanguardia, 13/10/2005) y produjo importantes afectaciones en las comunicaciones viarias en Barcelona, así como el cierre de algunos quirófanos en el Hospital Vall d'Hebron a causa de las filtraciones de agua, más de 150 salidas de los bomberos y la interrupción del servicio de la L5 del metro.

\section{Figura 6. Mapa de superficie y de $500 \mathrm{hPa}$ del episodio del 12/10/2005} $120 \mathrm{CT} 2005062$

500hPa Geopotential (gpdam), Bodendruck (hPa)

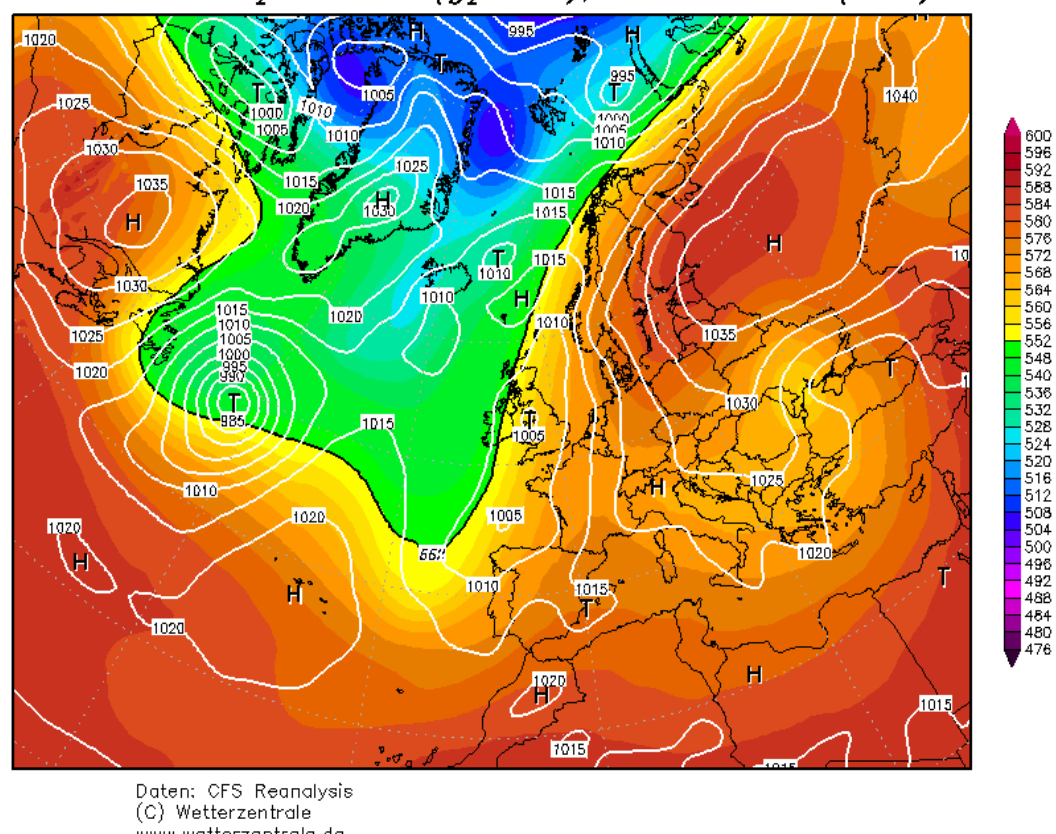

Fuente: Elaboración propia.

La Figura 7 muestra una fotografía en la que se puede ver la estación del metro de La Sagrera inundada. 


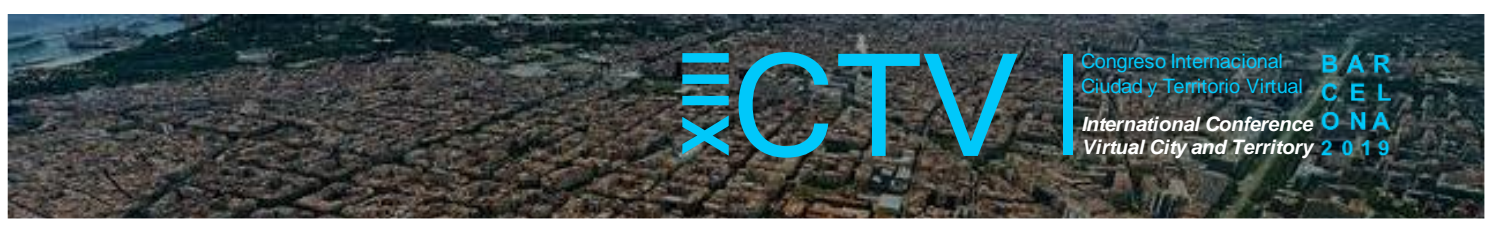

Figura 7. Estación del metro de La Sagrera inundada (La Vanguardia, 13/10/2005)

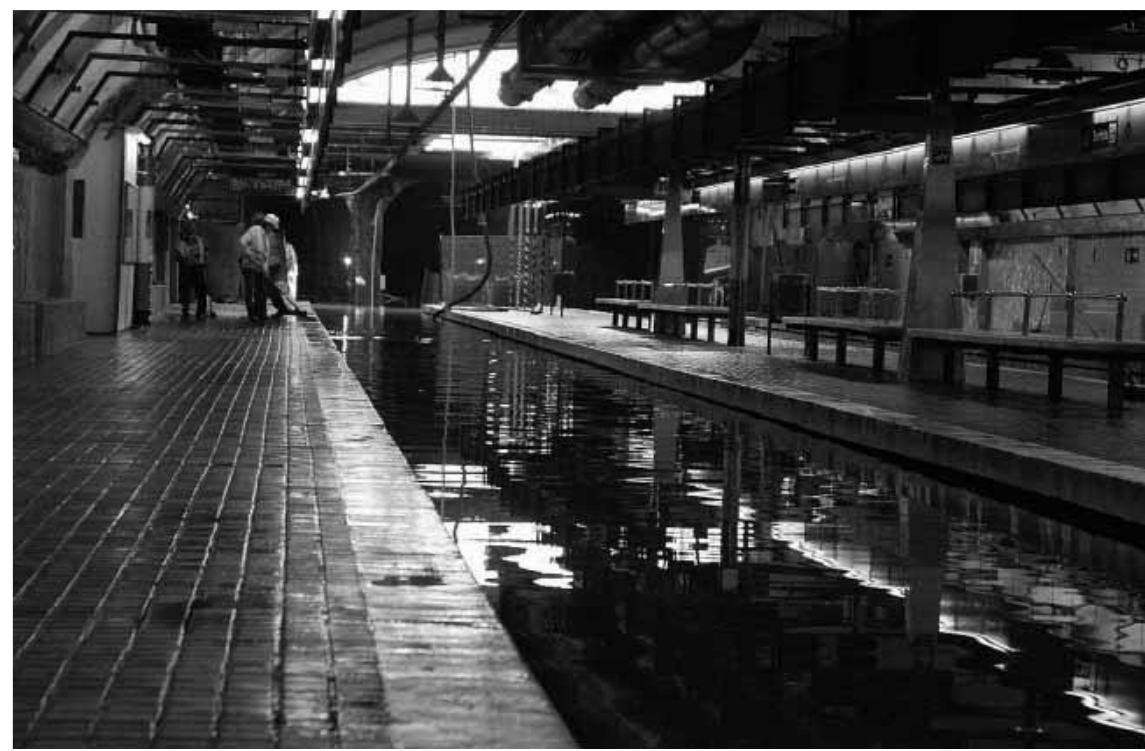

\section{Conclusiones}

En este trabajo, se han analizado los registros de intensidad de precipitación en Barcelona, proporcionados por la red pluviométrica de CLABSA para el periodo 1994-2009. Entre ellos, se han seleccionado los registros extremos, atendiendo a criterios basados en la cantidad total de precipitación acumulada, la intensidad máxima y la intensidad media. Estos registros se agrupan en siete episodios extremos de precipitación, todos ellos ocurridos entre finales de julio y octubre, que desbordaron la capacidad de los sistemas de drenaje y dieron lugar a inundaciones pluviales súbitas, provocando importantes afectaciones en la ciudad. Se han analizado también las distintas distribuciones temporales de la intensidad de lluvia a lo largo de los episodios, así como las situaciones sinópticas en las cuales se han producido estos episodios de precipitación copiosa en intervalos de tiempo relativamente cortos. El conocimiento de los patrones temporales y espaciales del régimen de precipitaciones intensas en las áreas urbanas constituye un elemento crucial para elaborar planes de actuación que permitan mitigar, en lo posible, el riesgo de inundaciones pluviales súbitas. El desarrollo de un planeamiento urbano dirigido a limitar la proporción de suelos sellados y la aplicación de medidas destinadas a mitigar y compensar, en lo posible, los efectos del sellado de los suelos serían piezas clave para reducir ese riesgo. Dado que el sellado de los suelos es un proceso casi irreversible, un objetivo del planeamiento debería ser limitarlo y, cuando ello no sea posible, usar suelos previamente desarrollados (zonas baldías, parcelas abandonadas), con el fin de preservar los suelos de mejor calidad (EC-DGENV, 2012). Por otra parte, los sistemas convencionales de gestión del agua de lluvia tienen como finalidad drenar rápidamente el agua, pero ello reduce la infiltración y la evaporación, lo cual tiene repercusiones negativas en la recarga de acuíferos y también en el efecto de isla de calor urbana. Además, estos sistemas pueden quedar desbordados, dando lugar a inundaciones súbitas, cuando se producen episodios de precipitación muy intensa, cuya frecuencia es probable que aumente como consecuencia del cambio climático y el calentamiento global (EEA, 2016). Durante los últimos años, se han desarrollado diversas técnicas de gestión sostenible de las aguas pluviales, que permiten reducir la escorrentía mediante un tratamiento lo más próximo posible al origen, a través de la captación del agua de lluvia y de un aumento de la infiltración y la evaporación. Un 


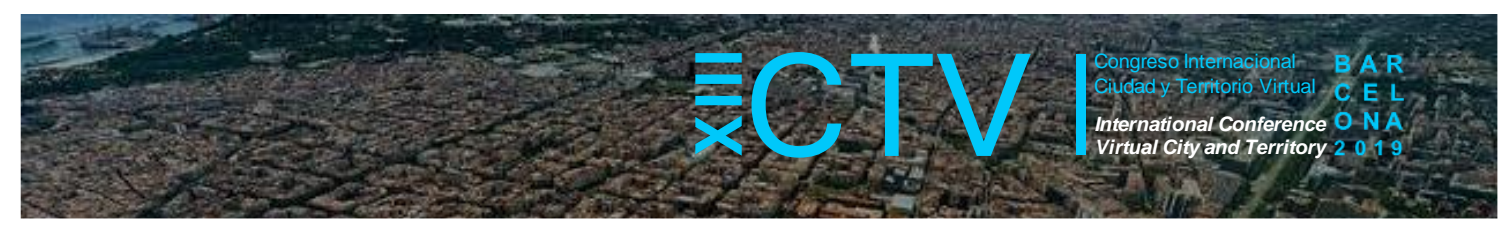

ejemplo es el planteamiento de WSUD (Water Sensitive Urban Design), que tiene como objetivo combinar las demandas de la gestión sostenible de las aguas pluviales con las demandas de la planificación urbana, acercando el ciclo urbano del agua al ciclo natural, mediante el desarrollo de estrategias integradoras para la sostenibilidad ecológica, económica, social y cultural (Hoyer et al., 2011).

Agradecimientos: Esta investigación ha sido financiada por el Ministerio de Economía y Competitividad (Gobierno de España), a través del proyecto BIA2015-68623-R. Los autores agradecen a CLABSA (Clavegueram de Barcelona, S.A.) los datos pluviométricos facilitados.

Conflicto de intereses: Los autores declaran que no hay conflicto de intereses.

\section{Bibliografía}

Casas, M. C.; Rodríguez, R.; Redaño, A. (2010) Analysis of extreme rainfall in Barcelona using a microscale rain gauge network. Meteorological Applications, 17,117-123. DOI https://doi.org/10.1002/met.166

EC. (2006). Communication from the Commission to the Council, the European Parliament, the European Economic and Social Committee and the Committee of the Regions - Thematic Strategy for Soil Protection, COM (2006) 231. Recuperado de http://eur-lex.europa.eu/legalcontent/EN/TXT/PDF/?uri=CELEX:52006DC0231\&from=EN

EC-DGENV. (2012). Commission Staff Working Document Guidelines on best practice to limit, mitigate or compensate soil sealing, European Commission, DG Environment, Brussels (SWD (2012) 101final/2). Recuperado de http://ec.europa.eu/environment/soil/pdf/guidelines/EN\%20$\% 20$ Sealing\%20Guidelines.pdf

EEA. (2014). Urban soil sealing in Europe, European Environment Agency. Recuperado de: http://www.eea.europa.eu/articles/urban-soil-sealing-in-europe

EEA. (2015). Urban vulnerability to climate change in Europe - A map book. European Environment Agency. Recuperado de http://climate-adapt.eea.europa.eu/tools/urbanadaptation/introduction

EEA. (2016). Climate change, impacts and vulnerability in Europe. European Environment Agency.

Findell, K. L.; Shevliakova, E.; Milly, P. C. D; Stouffer, R. J. (2007). Modelled impact of anthropogenic land cover change on climate. Journal of Climate, 20, 3621-3634. DOI https://doi.org/10.1175/JCLI4185.1

Giannaros, T. M.; Melas, D. (2012). Study of the urban heat island in a coastal Mediterranean City: The case study of Thessaloniki, Greece. Atmospheric Research, 118, 103-120. DOI https://doi.org/10.1016/j.atmosres.2012.06.006

Heusinkveld, B. G.; Steeneveld, G. J.; van Hove, L. W. A.; Jacobs, C. M. J.; Holtslag, A. A. M. (2014). Spatial variability of the Rotterdam urban heat island as influenced by urban land use. JGR: Atmospheres 119, 677-692. DOI https://doi.org/10.1002/2012JD019399 


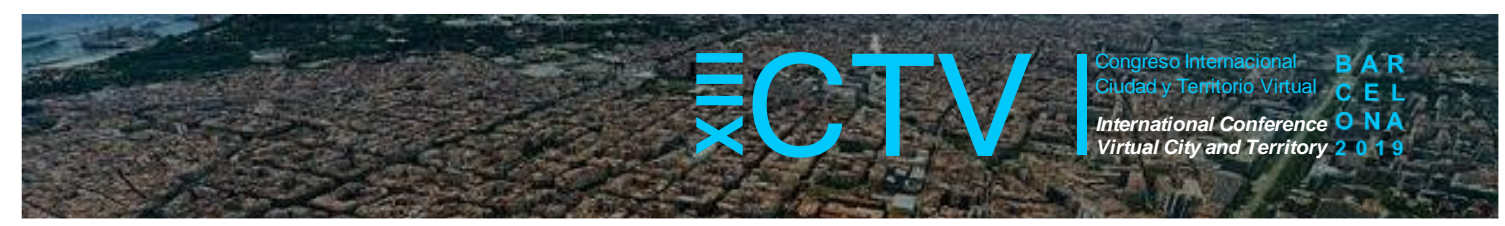

Hoyer, J.; Dickhaut, W.; Kronawitter, L.; Weber, B. (2011). Water Sensitive Urban Design Principles and Inspiration for Sustainable Stormwater Management in the City of the Future. Verlag GmbH, Kurfürstenstraße 15/16, Berlin, 115 pp.

Huff, F. A. (1967). Time distribution of rainfall in heavy storms. Water Resources Research, 3(4), 1007-1019. DOI https://doi.org/10.1029/WR003i004p01007

Kaźmierczak, A.; Cavan, G. (2011). Surface water flooding risk to urban communities: Analysis of vulnerability, hazard and exposure. Landscape and Urban Planning, 103(2), 185-197. DOI https://doi.org/10.1016/j.landurbplan.2011.07.008

Lana, X.; Serra, C.; Casas-Castillo, M. C.; Rodríguez-Solà, R.; Redaño, A.; Burgueño, A. (2018). Rainfall intensity patterns derived from the urban network of Barcelona (NE Spain). Theoretical and Applied Climatology, 133, 385-403. DOI https://doi.org/10.1007/s00704-017$\underline{2193-7}$

Lana, X.; Casas-Castillo, M. C.; Serra, C.; Rodríguez-Solà, R.; Redaño, A.; Burgueño, A.; Martínez, M. D. (2019a) Return period curves for extreme 5-min rainfall amounts at the Barcelona urban network. Theoretical and Applied Climatology, 135, 1243-1257. DOI https://doi.org/10.1007/s00704-018-2434-4

Lana, X.; Rodríguez-Solà, R.; Martínez, M. D.; Casas-Castillo, M. C.; Serra, C.; Burgueño, A. (2019b). Characterization of heavy rainfall profiles for Barcelona city: clustering, rain amounts and intensity peaks. Theoretical and Applied Climatology (submitted)

Makar, P. A.; Gravel, S.; Chirkov, V.; Strawbridge, K. B.; Froude, F.; Arnold, J.; Brook, J. (2006). Heat flux, urban properties, and regional weather. Atmospheric Environment, 40, 2750-2766. DOI https://doi.org/10.1016/i.atmosenv.2005.11.061

Martinez, M. D.; Lana, X.; Serra, C.; Roca, J.; Arellano, B.; Biere, R.; Moix, M. (2018). Características de la isla de calor urbana en Barcelona (NE España) en el periodo 2006-2017. En Libro de proceedings, CTV 2018. XII Congreso Internacional Ciudad y Territorio Virtual. "Ciudades y Territorios Inteligentes". UNCuyo, Mendoza, 5-7 septiembre 2018. Barcelona: CPSV, p. 369-380. DOI https://doi.org/10.5821/ctv.8252

Memon, R. A.; Leung, D. Y. C.; Chun-Ho Liu, C. (2009). An investigation of urban heat island intensity (UHII) as an indicator of urban heating. Atmospheric Research, 94, 491-500. DOI https://doi.org/10.1016/i.atmosres.2009.07.006

Oke, T. R. (1982). The energetic basis of the urban heat island. Quarterly Journal of the Royal Meteorological Society, 108, 1-24. DOI: https://doi.org/10.1002/qi.49710845502

Prokop, G.; Jobstmann, H.; Schönbauer, A. (2011). Overview on best practices for limiting soil sealing and mitigating its effects in EU-27, Environment Agency Austria. 